\title{
Homocysteine promotes cardiac fibrosis by regulating the Akt/ FoxO3 pathway
}

\author{
Ying Shi ${ }^{1,2 \#}$, Lili Zhao ${ }^{1 \#}$, Yifei Zhang ${ }^{3}$, Qin Qin ${ }^{1}$, Hongliang Cong ${ }^{1}$, Zhigang Guo ${ }^{4}$ \\ ${ }^{1}$ Tianjin Institute of Cardiovascular Disease, Tianjin Chest Hospital, Tianjin, China; ${ }^{2}$ Key Laboratory of Immune Microenvironment and Disease \\ (Tianjin Medical University), Ministry of Education, Tianjin, China; ${ }^{3}$ Academy of Medical Engineering and Translational Medicine, Medical \\ College, Tianjin University, Tianjin, China; ${ }^{4}$ Department of Cardiovascular Surgery, Tianjin Chest Hospital, Tianjin, China \\ Contributions: (I) Conception and design: Y Shi, L Zhao, Z Guo; (II) Administrative support: H Cong, Z Guo; (III) Provision of study materials or \\ patients: Y Shi, L Zhao, Y Zhang; (IV) Collection and assembly of data: Y Shi, L Zhao; (V) Data analysis and interpretation: Y Shi, L Zhao, Y Zhang, \\ Q Qin; (VI) Manuscript writing: All authors; (VII) Final approval of manuscript: All authors. \\ \#These authors contributed equally to this work. \\ Correspondence to: Hongliang Cong. Tianjin Institute of Cardiovascular Disease, Tianjin Chest Hospital, 261 Tai erzhuang Road, Jinnan District, \\ Tianjin 300222, China. Email: hongliangcong@163.com; Zhigang Guo. Department of Cardiovascular Surgery, Tianjin Chest Hospital, 261 Tai \\ erzhuang Road, Jinnan District, Tianjin 300222, China. Email: Zhigangguo@yahoo.com.
}

Background: Evaluated plasma homocysteine (Hcy) is an independent risk factor for cardiac fibrosis which
is a common feature of cardiovascular disease, although the mechanisms are still unclear. This study aims to
explore the mechanism of Hcy-induced cardiac fibrosis. Methods: The mRNA and protein levels of Forkhead box O3 (FoxO3) and differentiation markers were detected in primary cardiac fibroblasts (CFs) after $300 \mu M$ Hcy treatment. Scratch and transwell migration assay were used to determine the effect of Hcy on proliferation and migration in CFs. The protein levels involved in the fibrotic processes in mice fed with high methionine diet (HMD) for 4 or 8 weeks were investigated by western blot. CFs were infected with FoxO3 recombinant adenovirus to explore the potential role of FoxO3 in Hcy-induced cardiac dysfunction.

Results: Hcy treatment significantly promoted the differentiation, proliferation and migration of CFs, while FoxO3 activity were decreased in CFs. In HMD hearts, the protein levels of TIMP1, Fibronectin and $\alpha$-SMA were increased after 4 or 8 weeks, but the FoxO3 activity was decreased. Moreover, the HMD hearts had a higher level of $\mathrm{Bcl} 2$ but lower of Bax and LC3II protein. In addition, FoxO3 overexpression attenuates Hcy-induced dysfunction in CFs.

Conclusions: Hcy promotes myofibroblast activation and resistance to autophagy and apoptosis in CFs, and eventually results in cardiac fibrosis by regulating the $\mathrm{Akt} / \mathrm{FoxO} 3$ pathway. Thus, FoxO3 is a promising therapeutic target to prevent cardiac remodeling.

Keywords: Homocysteine (Hcy); forkhead box O3 (FoxO3); cardiac fibrosis; fibroblasts

Submitted Oct 08, 2021. Accepted for publication Nov 10, 2021.

doi: $10.21037 / \mathrm{atm}-21-5602$

View this article at: https://dx.doi.org/10.21037/atm-21-5602

\section{Introduction}

Cardiac fibrosis, a common pathological process involved in many forms of heart disease, is characterized by deposition of excessive extracellular matrix (ECM) proteins. Fibroblasts are the key effector cells in cardiac fibrosis. In response to injury or stress, cardiac fibroblasts (CFs) differentiate into myofibroblasts with increased expression of alpha-smooth muscle actin ( $\alpha$-SMA) and secretion of ECM proteins, especially collagen (1). Activated fibroblasts display hyperproliferative potential and abnormal active secretory activity (2). Although in the injured myocardium, expansion 
and activation of fibroblasts is crucial for normal healing and repair, CFs may also contribute to cardiac fibrosis, remodeling, and dysfunction (3).

Homocysteine (Hcy) is a non-essential sulfur-containing amino acid, which is formed via the classical remethylation and transsulfuration pathways. Hyperhomocysteinemia (HHcy) is diagnosed when plasma Hcy level is greater than $15 \mu \mathrm{mol} / \mathrm{L}$ (4). It has been established that evaluated plasma Hcy is an independent risk factor for many cardiovascular diseases (CVD), such as atherosclerosis (5), hypertension (6), and heart failure (7). Some studies have reported that high level of Hcy can enhance the transforming growth factor- $\beta$ (TGF- $\beta$ ) level and promote myocardial ECM remodeling and fibrosis $(8,9)$. The accumulation of cardiac collagen has been observed in models of HHcy $(10,11)$. Besides, the balance of tissue inhibitor of metalloproteinases (TIMPs)/ matrix metalloproteinases (MMPs) plays a critical role in maintaining the homeostasis of myocardial ECM. It has been reported that imbalance in the MMP-9-TIMP-1 axis accelerates ECM remodeling and renal fibrosis during HHcy (12). In addition to ECM remodeling, increased oxidant stress and inflammation may play an important role in the mechanical effects of HHcy on cardiac fibrosis $(13,14)$. In addition, Hcy has been shown to damage the endothelium and impair NO bioavailability (15), which may lead to left ventricular (LV) diastolic dysfunction. Recent study reported that downregulating IncRNA MEG3 could alleviate HCY-induced CF inflammation and cardiac fibrosis (16). The protective effect of SIRT1 on Hcyinduced atrial fibrosis has been reported and their results showed that the high-Hcy diet could promote the development of heart failure with preserved ejection fraction after transverse aortic constriction in mice (17). Despite large evidence for the involvement of Hcy in cardiovascular disease, our study aims to explore the precise molecular mechanisms responsible for Hcy-induced pathological changes in cardiac fibrosis, especially CFs.

Forkhead box O3 (FoxO3) is a member of the forkhead box transcription factors of the $\mathrm{O}$ class with a conserved helix-loop-helix DNA-binding domain; it is involved in a variety of vital cellular processes including oxidative stress, DNA repair, apoptosis, metabolism, and cell cycle arrest (18). Although the existing data are controversial, FoxO3 plays an important role in maintaining cardiovascular homeostasis. In cardiomyocytes, FoxO3 is able to inhibit cardiomyocyte hypertrophy (19) and promotes survival (20) through antioxidant activation. As a fibroblast regulator, FoxO3 is involved in renal, pulmonary, skin, liver, and cardiac fibrosis via regulation of fibroblast proliferation, differentiation, and apoptosis (21).

In this study, it was shown that $\mathrm{FoxO} 3$ plays a crucial role in Hcy-induced cardiac fibrosis. We found that $\mathrm{FoxO} 3$ was downregulated in CFs exposed to Hcy and also in HHcy model mice induced by high methionine diet (HMD). Furthermore, this study revealed that Hcy promotes the proliferation and differentiation and inhibits the autophagy and apoptosis of CFs through activation of the Akt/FoxO3 pathway. We present the following article in accordance with the ARRIVE reporting checklist (available at https:// dx.doi.org/10.21037/atm-21-5602).

\section{Methods}

\section{Primary CF culture and characterization}

We isolated CFs from the hearts of six 2-3-day-old Wistar rats. Briefly, hearts were harvested and cut into $1 \mathrm{~mm}^{2}$ small pieces. The tissues were digested with $0.05 \%(\mathrm{w} / \mathrm{v})$ tryptase (Gibco, Amarillo, TX, USA) and 0.05\% (w/v) collagenase II (Worthington, Lakewood, NJ, USA) at $37{ }^{\circ} \mathrm{C}$ with gentle agitation. After 5 digestions, the supernatants were pooled and centrifuged for $5 \mathrm{~min}$ at 1,000 rpm. The cell suspensions were filtered through a $100 \mu \mathrm{m}$ cell strainer and plated in $100 \mathrm{~mm}$ dishes (Eppendorf, Hamburg, Germany) with Dulbecco's modified Eagle medium (Gibco, USA) supplemented with $10 \%$ fetal bovine serum (FBS; Gibco, USA) and $1 \%$ penicillin/streptomycin for $2 \mathrm{~h}$, and then the medium was replaced. The attached cells were considered to be CFs, which were identified by discoidin domain receptor 2 (DDR2) immunostaining. Cells before passage 5 were used for further study.

\section{Animals}

We fed 6-week-old male C57BL/6 mice with a control chow diet or HMD (2\%) for 4 or 8 weeks. Fouty mice were randomly divided into four groups: control and HMD group respectively for 4 or 8 weeks. All animals were deeply anesthetized with isoflurane prior to removal of their hearts. The investigation conformed to the Guide for the Care and Use of Laboratory Animals by the US National Institutes of Health (NIH Publication No. 85-23, updated 2011). All experimental protocols were approved by Institutional Animal Care and Use Committee of Tianjin University (No. TJUE-2020-174). A protocol was prepared before the study without registration. The animal model was 
kindly supported by Professor Liu Yao (Tianjin Medical University, Tianjin, China).

\section{Recombinant adenovirus infection}

The FoxO3 expression plasmid was purchased from Sinobiological company (Beijing, China) and subsequently constructed into pAdEasy-1 vector. The recombinant adenovirus vector was linearized by Pac I enzyme, packaged into the $293 \mathrm{~T}$ cells and then purified by Adeno- $X$ Adenovirus purification kit (Clontech, BD Biosciences). For adenovirus infection, the $\mathrm{CFs}$ at $80 \%$ confluence were infected with adenovirus-FoxO3 or control adenovirusGFP at a multiplicity of infection (MOI) of 50 for 48 or $24 \mathrm{~h}$ under drug treatment conditions.

\section{Real time and quantitative polymerase chain reaction}

Total RNA was extracted with TRIzol Reagent (Invitrogen, Carlsbad, CA, USA) according to the manufacturer's instructions. Complementary DNAs (cDNA) were synthesized using the Takara reverse transcription system (Takara, Shiga, Japan). Real time quantitative-polymerase chain reaction (RT-qPCR) was conducted using the Takara SYBR Green SuperMix on 7500 real-time PCR system (Applied Biosystems; ABI, Waltham, MA, USA). The relative expression levels of specific genes were normalized to glyceraldehyde 3-phosphate dehydrogenase (GAPDH) levels, calculated with the $2^{-\Delta \Delta \mathrm{CT}}$ method.

\section{Western blot}

Cells were lysed with radioimmunoprecipitation assay (RIPA) buffer added with protease and phosphatase inhibitor cocktails on ice for $30 \mathrm{~min}$ and centrifuged at $12,000 \mathrm{rpm}$ for $30 \mathrm{~min}$ at $4{ }^{\circ} \mathrm{C}$. Protein concentration in the supernatants was quantified using a bicinchoninic (BCA) kit (Solarbio, Beijing, China). We separated 30-50 $\mu \mathrm{g}$ proteins by $10 \%$ gels and electroblotted to polyvinylidene fluoride (PVDF) membranes (Millipore, Burlington, MA, USA). Proteins were detected using specific antibodies of GAPDH, $\alpha$-SMA (Proteintech, Wuhan, China), FoxO3, phospho-FoxO3, Akt and phospho-Akt (Cell Signaling Technology, CST, Danvers, MA, USA) overnight at $4{ }^{\circ} \mathrm{C}$. The membranes were then incubated with horseradish peroxidase (HRP)-conjugated secondary antibody (Proteintech, Wuhan, China). The signal was visualized using the Pierce ECL Western Blotting Substrate (Thermo
Fisher Scientific, Waltham, MA, USA).

\section{Immunofluorescence staining}

The CFs for immunostaining were grown on 4 well chamber slides until 70-80\% confluence was reached. Slides were fixed in $4 \%$ paraformaldehyde for $15 \mathrm{~min}$ and permeabilized in $0.1 \%$ Triton-X100 (Sigma Aldrich, St. Louis, MO, USA) for $5 \mathrm{~min}$ at room temperature. Non-specific proteins were blocked with $3 \%$ bovine serum albumin (BSA) for $30 \mathrm{~min}$ at room temperature. Primary antibodies for $\alpha$-SMA, collagen I (Abcam, USA), FoxO3, and DDR2 (Santa Cruz Biotechnology, Santa Cruz, CA, USA) were incubated overnight at $4{ }^{\circ} \mathrm{C}$. After washing unbound primary antibodies with PBS, secondary antibodies were incubated at 1:200 in blocking buffer at room temperature for $1 \mathrm{~h}$. Fluorescein isothiocyanate (FITC)-conjugated goat anti-mouse or rabbit antibody (Abcam, USA) were applied as secondary antibodies. Nuclei were stained by 4',6-diamidino-2-phenylindole (DAPI; Zhongshan, Beijing, China). Images were acquired using a fluorescence microscope (Nikon, Tokyo, Japan).

\section{Scratch assay}

In brief, confluent monolayera of CFs were scratched with micropipette tip after overnight serum starvation. The cells were then washed twice with PBS to remove cell debris and incubated for $24 \mathrm{~h}$. The closed wound areas were measured to quantitatively evaluate cell migration using ImageJ software (https://imagej.nih.gov/ij/download.html).

\section{Transwell migration assay}

A modified Boyden chamber was used for transwell migration assay. The CFs $\left(2 \times 10^{5}\right)$ were resuspended in $200 \mu \mathrm{L}$ serum-free medium, and transferred to the upper chamber $(8 \mu \mathrm{m}$ pore size, Millipore, USA). The lower chambers were filled with $600 \mu \mathrm{L}$ complete medium. After $6 \mathrm{~h}$, cells were removed from the top of the membrane with a cotton swab and the membrane was mounted with DAPI mounting medium. The migratory cell images were taken with inverted microscope (Nikon, Japan) and analyzed using ImageJ software.

\section{Cell Counting Kit-8 (CCK-8) assay}

After cells were cultured and treated, CCK-8 (Bioss, 
A
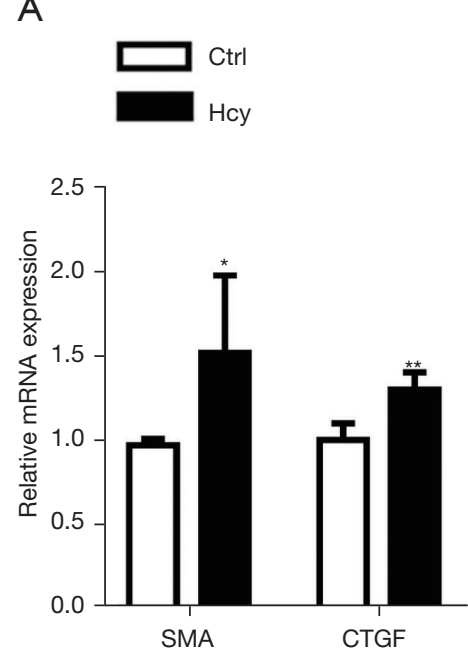

C

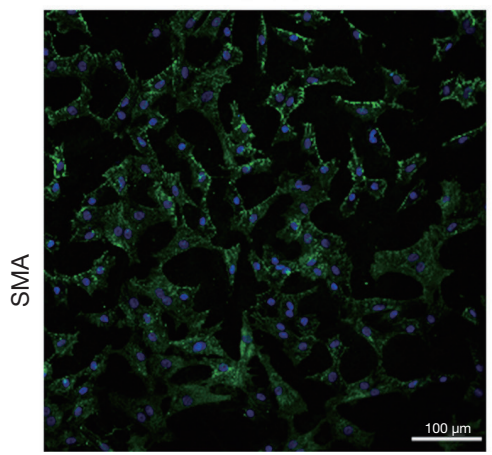

B
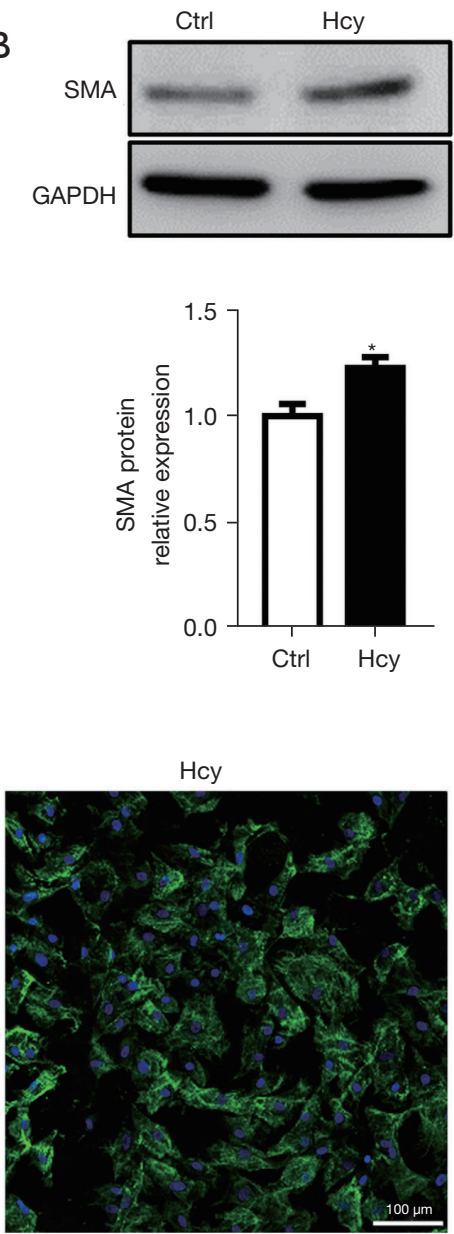

Figure $1 \mathrm{Hcy}$ induces fibroblasts differentiation into myofibroblasts. Neonatal rat cardiac fibroblasts (NRCFs) were treated with Homocysteine (Hcy) $(300 \mu \mathrm{M})$ for $24 \mathrm{~h}$. (A) The mRNA levels of smooth muscle actin (SMA) and CTGF (connective tissue growth factor) were detected by qRT-PCR (quantitative polymerase chain reaction), n=3-5. (B) Western blotting (up) and quantification (down) for SMA protein, $\mathrm{n}=3$. (C) Representative immunostaining of SMA protein, Scale bar, $100 \mu \mathrm{m}$. All data are shown as means \pm SEM, unpaired $t$-test was used for comparisons. *, $\mathrm{P}<0.05 ;{ }^{* *}, \mathrm{P}<0.01$.

Wuhan, China) was added and incubated for $2 \mathrm{~h}$. The optical density (OD) at $450 \mathrm{~nm}$ was detected. Cell viability was calculated as cell viability $=\mathrm{OD}$ (treatments)/OD (controls) $100 \%$.

\section{Statistical analysis}

All data were expressed as mean \pm standard error of the mean (SEM). One-Way analysis of variance (ANOVA) was used for multigroup comparison. Student's $t$-test was used for two group comparison. Values of $\mathrm{P}<0.05$ were considered statistically significant. Statistical analyses and graphics were carried out with GraphPad Prism 8 software (GraphPad Software, San Diego, CA, USA).

\section{Results}

\section{Hcy induced fibroblasts differentiation into myofibroblasts}

To study the role of Hcy, we isolated primary CFs from newborn rat hearts as described previously (22) and identified them by flat-spindle shape and expressing DDR2 (Figure S1). The differentiation of fibroblasts into myofibrolasts is the important process during cardiac fibrosis. Thus, we detected the levels of differentiation markers, such as $\alpha$-SMA and connective tissue growth factor (CTGF). Results showed that CFs displayed a significantly increased messenger RNA (mRNA) expression after exposure to $300 \mu \mathrm{M}$ Hcy (Figure 1A). Similarly, the protein level of $\alpha$-SMA was significantly upregulated by high level 


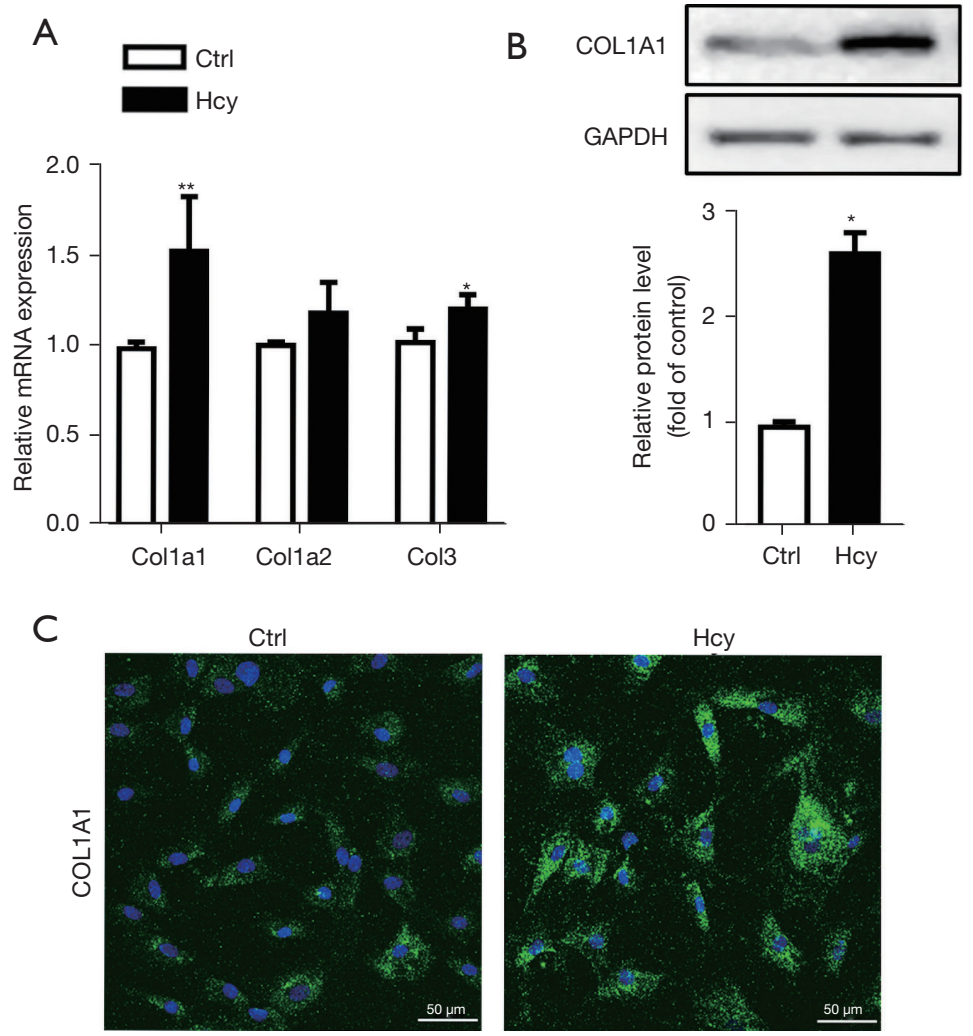

Figure 2 Hcy induces collagen synthesis in cardiac fibroblasts. (A) qPCR analysis of col1a1, col1a2, col3 (collagen), n=3-5. (B) Western blot analysis (up) and quantification (down) of the expression of COL1A1 protein, $n=3$. (C) Representative immunostaining of COL1A1, Scale bar, $50 \mu \mathrm{m}$. All data are shown as means $\pm \mathrm{SEM}$, unpaired $t$ test was used for comparisons, ${ }^{*}, \mathrm{P}<0.05 ;{ }^{* *}, \mathrm{P}<0.01$.

of Hcy (Figure 1B,1C).

\section{Hcy promotes the remodeling of ECM}

Next, we assessed the effect of Hcy on myocardial ECM. Type I collagen, the most important and main component of fibrosis, is synthesized and secreted mostly by CFs (23). We observed a significant increase of Collagen 1a1 (COL1A1) and Collagen 3 (COL3) mRNA levels in Hcy treated CFs (Figure $2 A$ ), however, there were no significant differences of Colla2 between Ctrl and Hcy group. Consistent with the RNA analysis, high level of Hcy caused a significant increase in COL1A1 protein level (Figure 2B,2C). Moreover, the ED-A domain of fibronectin is involved in the activation of myofibroblast (24). Our data also showed that Hcy treatment significantly enhanced the mRNA and protein levels of fibronectin (Figure $3 A-3 D$ ). In a similar way, we found that the protein levels of TIMP1 were increased in Hcy treated CFs with no change in RNA levels (Figure $3 E-3 H$ ). It has been reported that TIMP1 directly promotes myocardial fibrosis and induced collagen synthesis in CFs (25). Together, our data indicate that Hcy promotes myocardial fibrosis through fibroblast activation and ECM expansion.

\section{Hcy promotes the proliferation and migration of CFs}

In determining the influence of Hcy on the growth of fibroblasts, the result of CCK-8 assay showed that Hcy significantly promoted the CFs proliferation (Figure S2). The cytokine TGF- $\beta$ is the most well-characterized profibrotic cytokine that triggers fibroblast activation both in vitro and in vivo. As a positive control, TGF- $\beta$ treatment strongly increased the migratory capacity and proliferation as compared to untreated cells in the scratch and transwell assay. Importantly, Hcy treatment also promoted the proliferation and migration of CFs similarly to TGF- $\beta$ (Figure 4A-4D).

\section{FoxO3 participates in Hcy-mediated effects in CFs}

We next investigated the possible mechanism that Hcy 


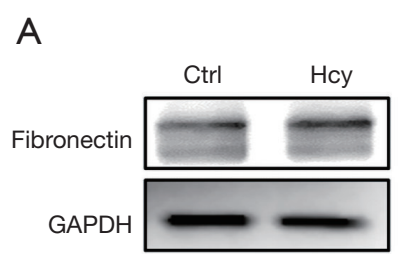

B

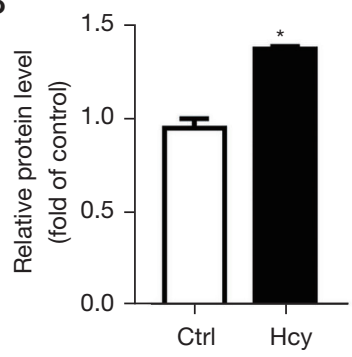

$E$

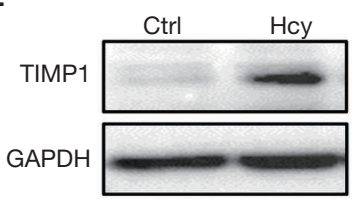

$\mathrm{F}$

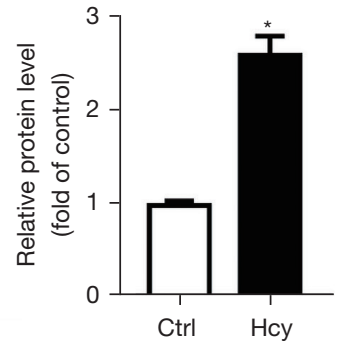

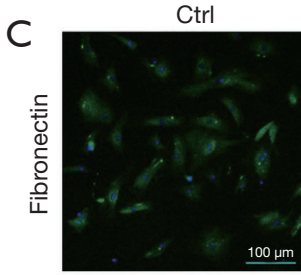

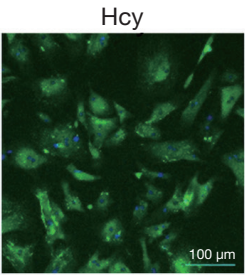

D
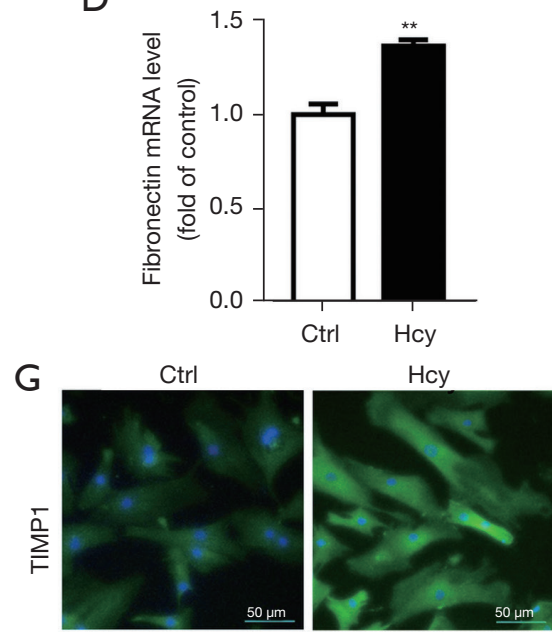

$\mathrm{H}$

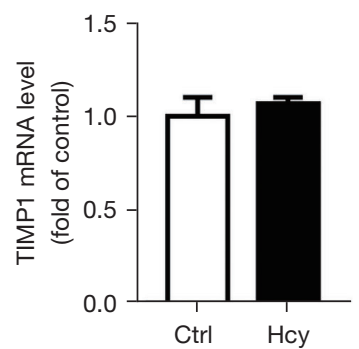

Figure 3 Hcy promotes the remodeling of extracellular matrix in cardiac fibroblasts. (A) Western blot analysis and quantification (B) of the expression of Fibronectin protein, n=3. (C) Representative immunofluorescence micrographs of fibronectin (FN), scale bar, 100 $\mu \mathrm{m}$. (D) qPCR analysis of fibronectin, $n=3-5$. (E) Western blot analysis and quantification (F) of the expression of tissue inhibitor of metalloproteinase 1 (TIMP1) protein, $\mathrm{n}=3$. (G) Representative immunofluorescence micrographs of TIMP1, Scale bar, $50 \mu \mathrm{m}$. (H) qPCR analysis of TIMP1, $\mathrm{n}=3-5$. All data are shown as means $\pm \mathrm{SEM}$, unpaired $t$ test was used for comparisons. ${ }^{*}, \mathrm{P}<0.05 ;{ }^{* *}, \mathrm{P}<0.01$.

promotes the activation of CFs. Having shown that FoxO3 regulates cardiac fibrosis (26), we found that the mRNA expression of FoxO3 was greatly downregulated in fibroblasts exposed to Hcy (Figure $5 A$ ). It has been shown that FoxO3 can be phosphorylated at T32, S253, and S315 residues through the phosphatidylinositol3-kinase (PI3K)/Akt signaling pathway (27). We demonstrated that the relative phosphorylation of FoxO3 at Ser253 was significantly increased accompanied by remarkably increased Akt phosphorylation (Figure 5B-5D). These data suggest that FoxO3 activity is suppressed by Hcy exposure in CFs. Phosphorylation of FoxO3 usually causes its exclusion from the nucleus and suppresses its transcriptional activity (24). As shown in Figure 5E, Hcy-treated CFs exhibited decreased levels of nuclear and total FoxO3. Overall, these results confirm the idea that Hcy drives FoxO3 downregulation in CFs.

\section{FoxO3 ameliorates the Hcy-induced dysfunction of CFs}

To assess whether the effects of Hcy are mediated via FoxO3, we infected the $\mathrm{CF}$ s with recombinant adenovirus to overexpress FoxO3. Previous studies have shown that FoxO3 promotes the cellular apoptosis (28) and autophagy (29) 

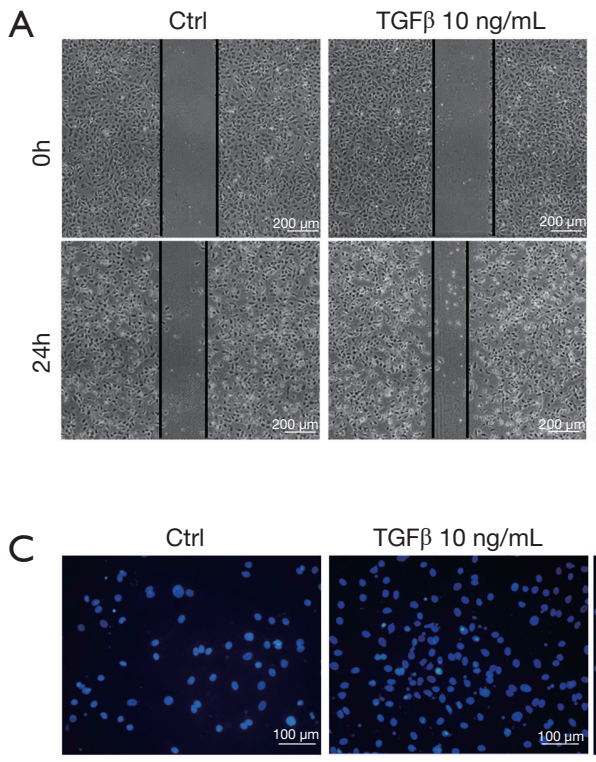

Hcy $300 \mu \mathrm{M}$

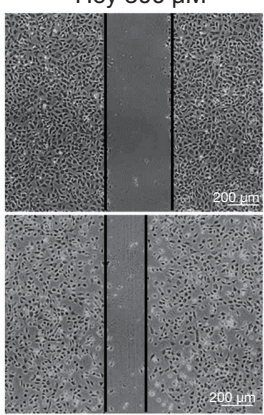

Hсу $300 \mu \mathrm{M}$

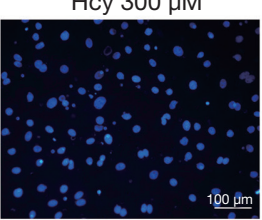

B
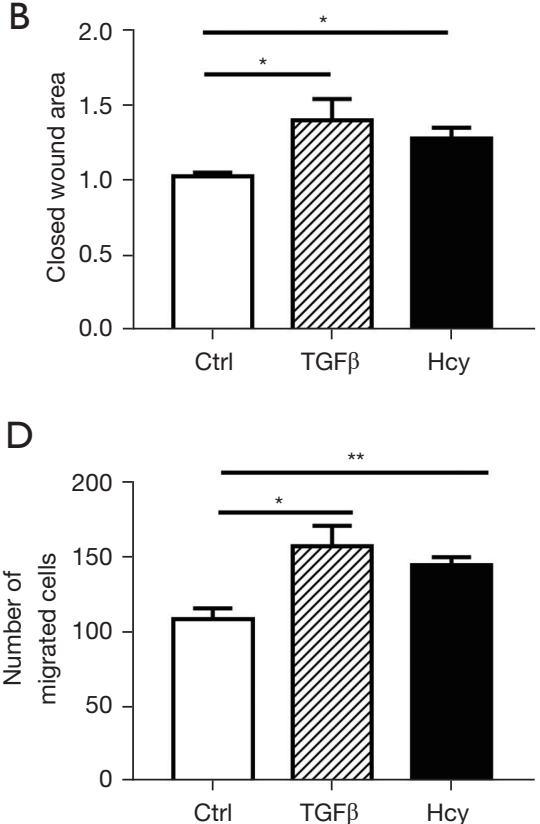

Figure 4 Hcy promotes the proliferation and migration of cardiac fibroblasts. (A) Scratch assay in cardiac fibroblasts followed by transforming growth factor- $\beta$ (TGF- $\beta$ ) $(10 \mathrm{ng} / \mathrm{mL})$ or $300 \mu \mathrm{M} \mathrm{Hcy}$; images were photoed at 0 and $24 \mathrm{~h}$ after scratch. Black lines illustrate the wound borders. Scale bar, $200 \mu \mathrm{m}$; (B) quantification of the closed wound area, $n=4$; (C) transwell assay of migrating cells and quantification; (D) cell numbers from 7-8 random fields were counted in each well. Scale bar, $100 \mu \mathrm{m}, \mathrm{n}=3-5$. Data are shown as means \pm SEM, unpaired $t$ test was used for comparisons. *, $\mathrm{P}<0.05 ;{ }^{* *}, \mathrm{P}<0.01$.

but inhibits proliferation (30). Consistent with these notions, western blot results (Figure $5 F$ ) showed that overexpression of $\mathrm{FoxO} 3$ decreased Hcy-induced expression of $\mathrm{Bcl} 2$ protein, but increased the expression of Bax (Figure 5G,5H). In addition, overexpressing $\mathrm{FoxO} 3$ increased Hcy-induced inhibition of LC3II expression (Figure 5I). Furthermore, $\mathrm{FoxO} 3$ overexpression significantly decreased a-SMA protein level which was enhanced by Hcy treatment (Figure 57); however, the COL1A1 expression was not significantly changed (result not shown). In contrast, we treated CFs with small interfering RNA (siRNA) to silence FoxO3 and the FoxO3-silenced CFs showed greatly increased mRNA levels of $\alpha$-SMA and col1a1 (Figure S3). These findings suggest that the Hcy-induced dysfunction of CFs is partly mediated via decreased $\mathrm{FoxO} 3$ activity.

\section{Hcy promotes cardiac remodeling through the Akt/FoxO3 pathway in mice}

To ascertain the role of Hcy on fibrotic processes in vivo, mice were fed an HMD for 4 or 8 weeks (31). In HMD hearts, the protein levels of TIMP1, fibronectin, and $\alpha$-SMA were increased after 4 and 8 weeks (Figure $6 A-6 D$ ), indicating that HHcy promoted myocardial remodeling and fibrosis. In addition, to determine whether the profibrotic effect of $\mathrm{Hcy}$ is accompanied by decreased $\mathrm{FoxO} 3$ activity, we assessed the status of FoxO3 phosphorylation in HMD hearts. A significant increase of FoxO3 phosphorylation at Ser253 was observed in HMD-fed mice compared with chow-fed mice. Further, we examined the role of PI3K/Akt pathway in this process. Similarly, HMD-induced HHcy significantly enhanced the levels of phosphorylated Akt (Figure 6A-6D).

We next evaluated the expression of apoptosis and autophagy related protein, and western blot results showed that HMD hearts had a higher level of Bcl2 but lower level of Bax and LC3II protein (Figure 6E-67), indicating that Hcy resulted in resistance to apoptosis and autophagy, in agreement with previous findings. Collectively, these data suggest that Hcy promotes cardiac remodeling through the Akt/FoxO3 axis.

\section{Discussion}

Fibroblasts are the major resource of cardiac remodeling. 
A

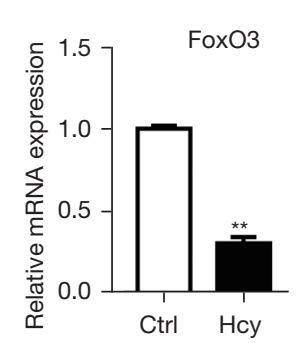

B

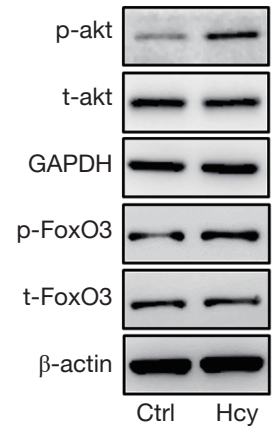

$\mathrm{F}$

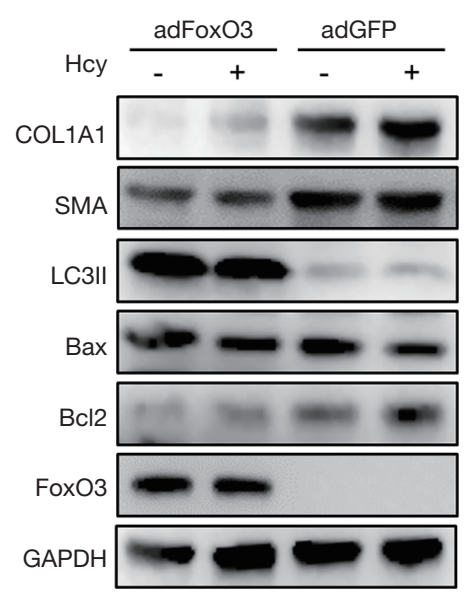

C

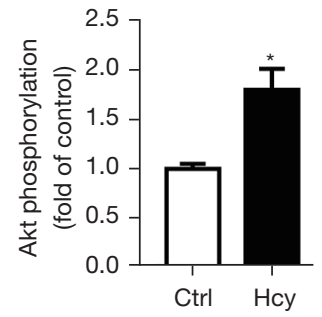

D

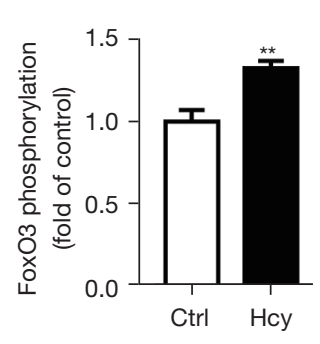

$\mathrm{E}$
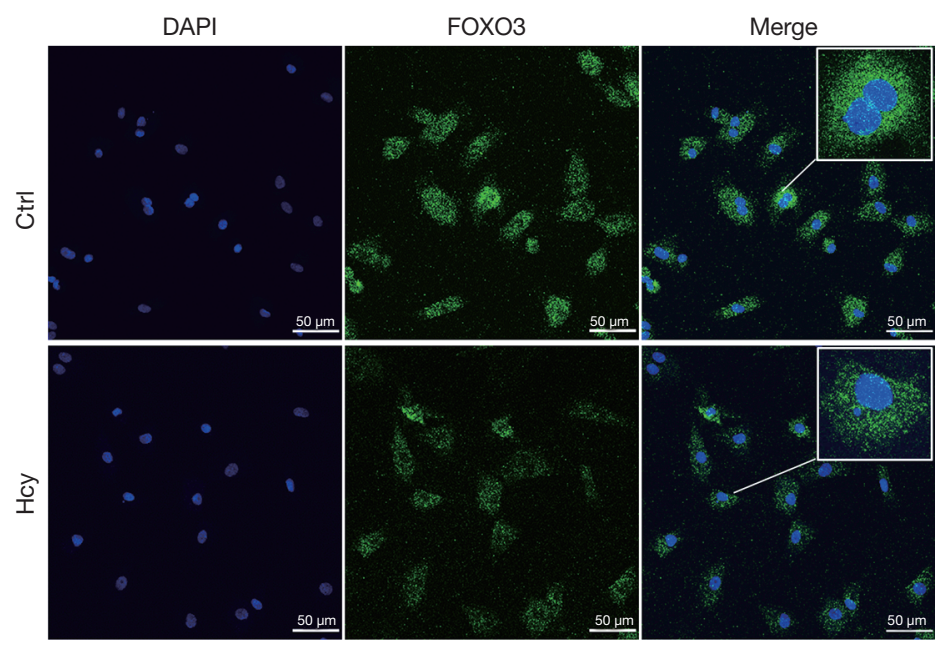

$\mathrm{H}$

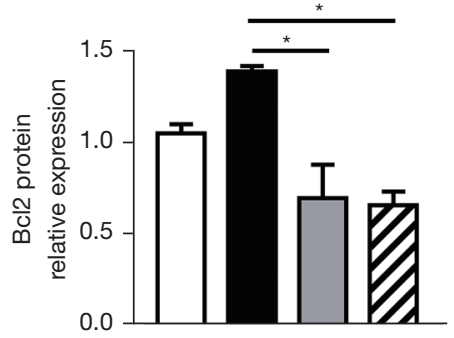

I

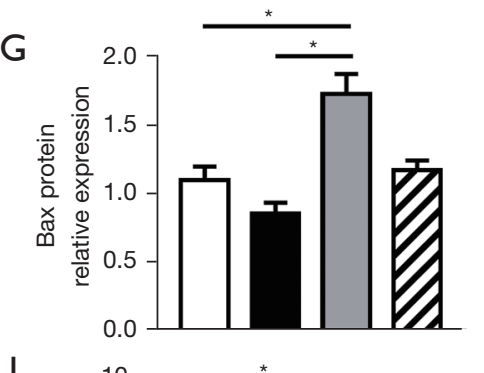

J

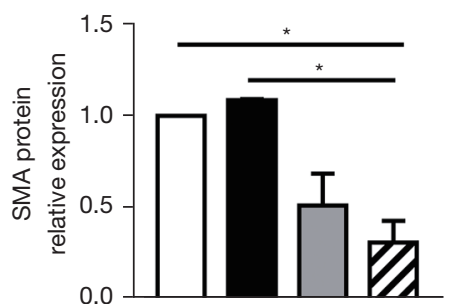

Figure 5 FoxO3 ameliorates the Hcy-induced dysfunction of cardiac fibroblasts. Cardiac fibroblasts (CFs) were treated with Homocysteine (Hcy) for 24 h. (A) qPCR analysis of FoxO3 mRNA levels, n=5; (B) western blot analysis and quantification (C and D) of Akt and FoxO3 phosphorylation levels in CFs after Hcy treatment, n=3; (E) Representative immunostaining of FoxO3 protein, Scale bar, $50 \mu \mathrm{m}$. Then CFs were infected with ad-GFP or ad-FoxO3 for $24 \mathrm{~h}$ followed by Hcy for another $24 \mathrm{~h}$. (F) Western blot analysis and quantification (G-J) of SMA, LC3II, Bax, Bcl2 and FoxO3 protein, $\mathrm{n}=3$; Data are shown as means \pm SEM, 1-way ANOVA was used for comparisons. *, $\mathrm{P}<0.05$; **, $\mathrm{P}<0.01$.

They are quiescent in normal healthy hearts and become activated and converted into myofibroblasts after injury. Increasing evidence has demonstrated that increased level of plasma Hcy is associated with cardiac fibrosis (14). However, the precise mechanisms by which Hcy contributes to fibroblast dysfunction during cardiac fibrosis remain elusive. Here, we have reported the findings that Hcy promotes cardiac remodeling and dysfunction through the PI3K/Akt/FoxO3 pathway and FoxO3 alleviates Hcyinduced myofibroblast activation partly by inhibiting cellular apoptosis and autophagy.

Methionine, the only source of Hcy, can be metabolized 


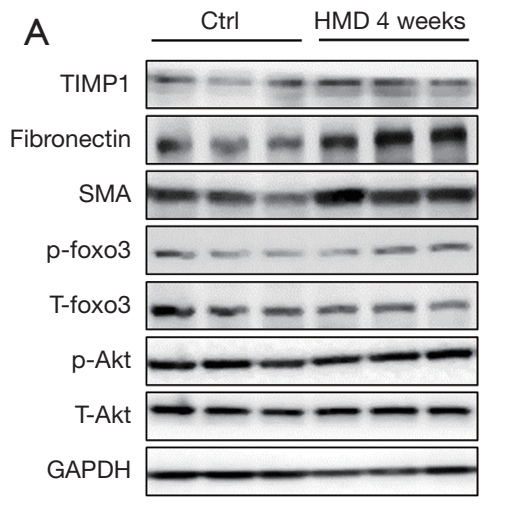

B

HMD 4 weeks
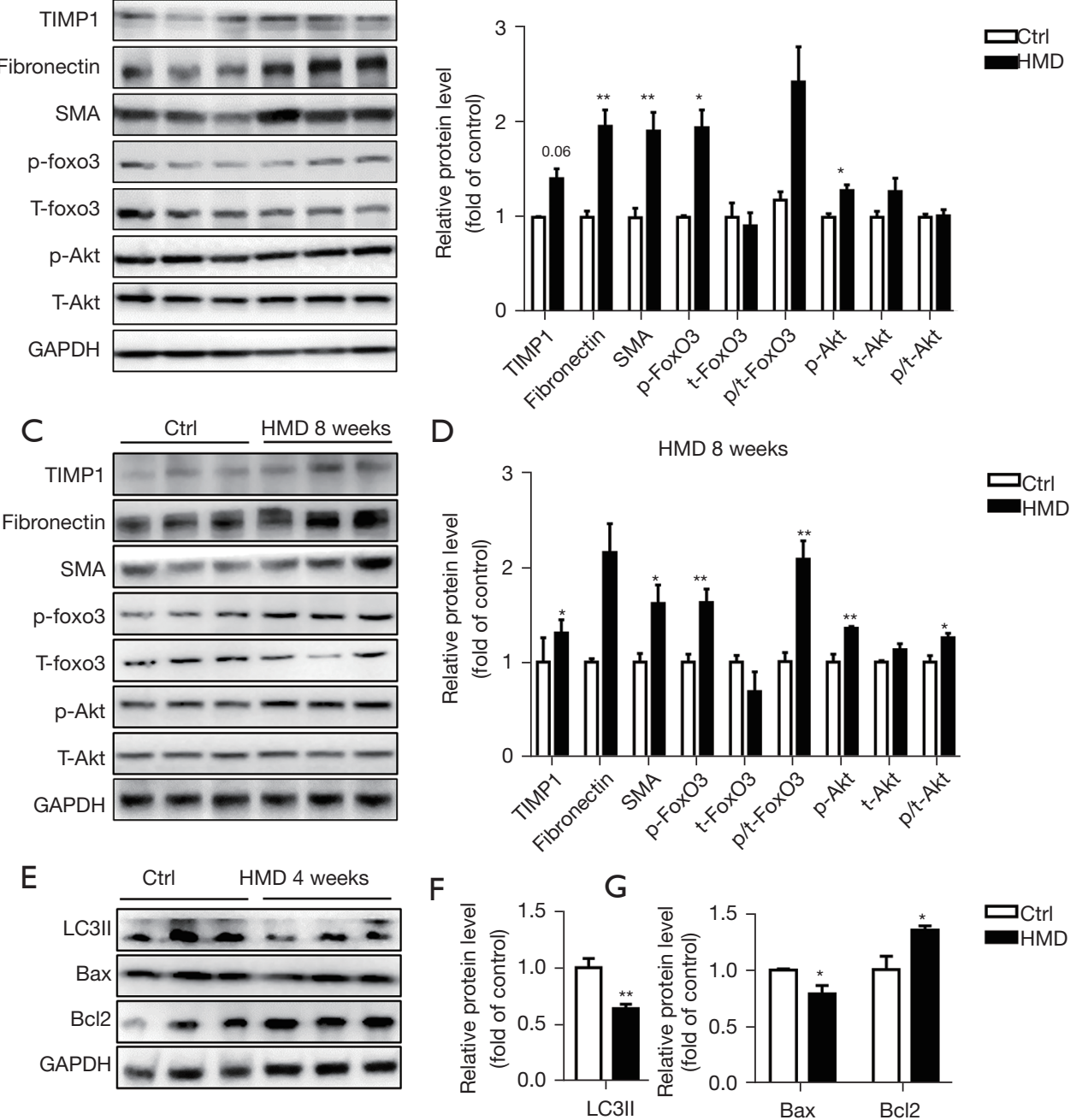

G
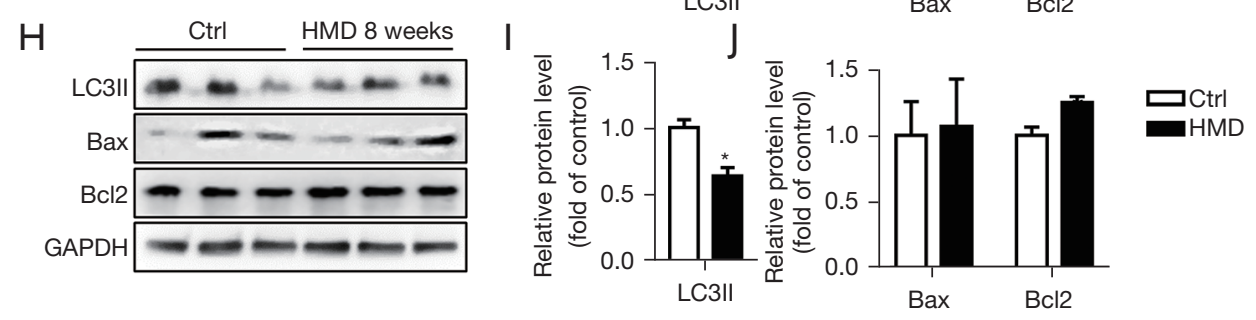

Figure 6 Hcy promotes cardiac remodeling through Akt/FoxO3 pathway in mice. The C57BL6 mice were fed with high methionine diet (HMD) for 4 or 8 weeks. The heart was separated and extracted proteins. (A-D) Western blot for TIMP1, Fibronectin, SMA, total and phosphorylated FoxO3 and total and phosphorylated Akt in hearts from mice after 4 weeks (A and B) or 8 weeks (C and D) HMD feeding and quantification, n=5. (E-J) Western blot analysis and quantification of LC3II, Bax and Bcl2 protein after 4 weeks (E-G) or 8 weeks (H-J) HMD feeding, $\mathrm{n}=5$. Data are shown as means $\pm \mathrm{SEM}$, unpaired $t$ test was used for comparisons. ${ }^{*}, \mathrm{P}<0.05$; ** $\mathrm{P}<0.01$.

through three pathways: the methionine cycle, the transsulfuration pathway, and the salvage cycle. Under the metabolism of a series of enzymes, methionine can be converted to Hcy. Dysregulation of methionine metabolism has been reported in multiple diseases, such as heart, liver and kidneys. High methionine diet induced oxidative stress, inflammation, and vascular remodeling, finally resulted in decreased cardiac function in mice (32). Increasing evidence 
suggests methionine restriction (MR) can prolong life (33), improve cardiac dysfunction (34) and other beneficial effects. In our study, Hcy promoted the conversion of fibroblasts to myofibroblasts expressing $\alpha$-SMA and induced proliferation and migration. The highly proliferative myofibroblasts secrete large amounts of ECM proteins. The deposition and expansion of ECM plays a crucial role in cardiac dysfunction (35). In this study, Hcy induced an enhancement in the expression of the pro-fibrotic markers such as Collagen1, fibronectin, and TIMP1 in vitro and in vivo. It is worth noting that fibronectin can not only form a matrix network but also facilitate myofibroblast differentiation (36). In addition, upregulated TIMP1 may act as a matrix-preserving factor, promoting ECM deposition and increasing myocardial stiffness (36). These data indicate that Hcy accelerates fibrotic progress. In general, a better understanding of the underlying molecular mechanisms of fibroblast dysfunction is important for the development of novel anti-fibrotic therapies.

Defective autophagy results in development of heart failure (37), however the role of autophagy in CFs is less known. Recent evidence indicated that autophagic flux was impaired in activated CFs and fibrotic hearts (38). Another study found that autophagy enhanced the activation of CFs and exerted a profibrotic role (39). The present study showed that Hcy caused a significant decrease in LC3II protein levels compared with normal controls in CFs and in mice. It confirmed that Hcy could accelerate the development of fibrosis through inhibition of autophagy. Interestingly, we found that Hcy results in downregulation of Bax but upregulation of Bcl2 in CFs. Our findings are completely opposite from results in $\mathrm{H} 9 \mathrm{c} 2$ cardiomyocytes (40). Others have demonstrated that HHcy could induce hypertrophy and apoptosis of cardiomyocytes, leading to cardiac fibrosis in mice fed with HMD for 12 weeks (41). In our study, however, the roles of Hcyinhibition of autophagy and apoptosis were observed in mice fed with HMD. We attribute this to the diverse functions of Hcy depending on different tissues and conditions. Apoptosis and autophagy are both evolutionarily conserved pathways that tightly regulate and interact with each other; anti-apoptotic Bcl-2 can inhibit autophagy via an interaction with Beclin1 (42). Our findings suggest that Hcy-induced Bcl2 might impede autophagy and result in myofibroblast prolonged persistence.

It is known that FoxO3 regulates several cellular functions such as apoptosis, cell cycle arrest, autophagy, oxidative stress resistance, and longevity (27). We propose that $\mathrm{FoxO} 3$ is involved in Hcy-induced cardiac remodeling. Our results showed that Hcy inhibits FoxO3 expression and nucleus location in fibroblasts. Furthermore, the PI3K/ Akt/FoxO3 pathway was activated in HMD-hearts. Most importantly, FoxO3 overexpression could reverse the effects of Hcy in CFs, evidenced by SMA downregulation and decreased Bcl2 expression but increased Bax. It is well known that activated fibroblasts are resistant to apoptosis (1). Thus, therapies that induce apoptosis of activated fibroblasts might be beneficial in progressive fibrotic diseases. On the other hand, autophagy may function as a defensive mechanism in CFs by autophagic degradation of intracellular collagen (43). We found that the Hcy-inhibited LC3II was upregulated by overexpressing FoxO3. These findings revealed that $\mathrm{FoxO} 3$ promotes the apoptosis and autophagy of CFs to protect against Hcy-induced cardiac fibrosis. Thus, future studies are warranted to determine the potential therapeutic benefit of $\mathrm{FoxO} 3$ upregulation in CFs for the prevention of cardiac remodeling.

In conclusion, we demonstrated that Hcy may reduce the autophagy and apoptosis of CFs and promote fibroblast activation, eventually resulting in cardiac fibrosis via activation of the PI3K/Akt/FoxO3 pathway. Therefore, FoxO3 agonists should be carefully evaluated as antifibrotic agents for treating cardiac remodeling in the future. However, there are some limitations in this study. We only investigated the role of FoxO3 in neonatal rat CFs, thus further study should be considered using knockout mice or adenoviral gene delivery to investigate the pathological changes and potential mechanism.

\section{Acknowledgments}

In this study, the animal model was kindly supported by Dr. Liu Yao (Tianjin Medical University, Tianjin, China).

Funding: This study was supported by the Natural Science Foundation of Tianjin City (20JCQNJC01980) and the Tianjin Haihe Medical Scholars Fund and the Open Foundation of the Key Laboratory of Immune Microenvironment and Disease (Tianjin Medical University), Ministry of Education (20190201) and Science foundation of Tianjin Chest Hospital (2018XKZ18).

\section{Footnote}

Reporting Checklist: The authors have completed the ARRIVE reporting checklist. Available at https://dx.doi. org/10.21037/atm-21-5602 
Data Sharing Statement: Available at https://dx.doi. org/10.21037/atm-21-5602

Conflicts of Interest: All authors have completed the ICMJE uniform disclosure form (available at https://dx.doi. org/10.21037/atm-21-5602). All authors report that this study was supported by the Natural Science Foundation of Tianjin City (20JCQNJC01980) and the Tianjin Haihe Medical Scholars Fund and the Open Foundation of the Key Laboratory of Immune Microenvironment, Disease (Tianjin Medical University) and Ministry of Education (20190201) and Science foundation of Tianjin Chest Hospital (2018XKZ18), and the payments were made to the institutions. The authors have no other conflicts of interest to declare.

Ethical Statement: The authors are accountable for all aspects of the work in ensuring that questions related to the accuracy or integrity of any part of the work are appropriately investigated and resolved. All experimental protocols were approved by Institutional Animal Care and Use Committee of Tianjin University (No. TJUE2020-174). The investigation conformed to the Guide for the Care and Use of Laboratory Animals by the US National Institutes of Health (NIH Publication No. 8523, updated 2011).

Open Access Statement: This is an Open Access article distributed in accordance with the Creative Commons Attribution-NonCommercial-NoDerivs 4.0 International License (CC BY-NC-ND 4.0), which permits the noncommercial replication and distribution of the article with the strict proviso that no changes or edits are made and the original work is properly cited (including links to both the formal publication through the relevant DOI and the license). See: https://creativecommons.org/licenses/by-ncnd/4.0/.

\section{References}

1. Gibb AA, Lazaropoulos MP, Elrod JW. Myofibroblasts and Fibrosis: Mitochondrial and Metabolic Control of Cellular Differentiation. Circ Res 2020;127:427-47.

2. Hinderer S, Schenke-Layland K. Cardiac fibrosis - A short review of causes and therapeutic strategies. Adv Drug Deliv Rev 2019;146:77-82.

3. Humeres C, Frangogiannis NG. Fibroblasts in the Infarcted, Remodeling, and Failing Heart. JACC Basic
Transl Sci 2019;4:449-67.

4. Zaric BL, Obradovic M, Bajic V, et al. Homocysteine and Hyperhomocysteinaemia. Curr Med Chem 2019;26:2948-61.

5. Antoniades C, Antonopoulos AS, Tousoulis D, et al. Homocysteine and coronary atherosclerosis: from folate fortification to the recent clinical trials. Eur Heart J 2009;30:6-15.

6. Dinavahi R, Falkner B. Relationship of homocysteine with cardiovascular disease and blood pressure. J Clin Hypertens (Greenwich) 2004;6:494-8; quiz 499-500.

7. Herrmann M, Taban-Shomal O, Hübner U, et al. A review of homocysteine and heart failure. Eur J Heart Fail 2006;8:571-6.

8. Jeremic J, Nikolic Turnic T, Zivkovic V, et al. Vitamin B complex mitigates cardiac dysfunction in high-methionine diet-induced hyperhomocysteinemia. Clin Exp Pharmacol Physiol 2018;45:683-93.

9. Raaf L, Noll C, Cherifi Mel H, et al. Myocardial fibrosis and TGFB expression in hyperhomocysteinemic rats. Mol Cell Biochem 2011;347:63-70.

10. Carroll JF, Tyagi SC. Extracellular matrix remodeling in the heart of the homocysteinemic obese rabbit. Am J Hypertens 2005;18:692-8.

11. Joseph J, Pencina MJ, Wang TJ, et al. Cross-sectional relations of multiple biomarkers representing distinct biological pathways to plasma markers of collagen metabolism in the community. J Hypertens 2009;27:1317-24.

12. Pushpakumar S, Kundu S, Narayanan N, et al. DNA hypermethylation in hyperhomocysteinemia contributes to abnormal extracellular matrix metabolism in the kidney. FASEB J 2015;29:4713-25.

13. Zhang JS, Hou YL, Lu WW, et al. Intermedin1-53 Protects Against Myocardial Fibrosis by Inhibiting Endoplasmic Reticulum Stress and Inflammation Induced by Homocysteine in Apolipoprotein E-Deficient Mice. J Atheroscler Thromb 2016;23:1294-306.

14. Kar S, Shahshahan HR, Kambis TN, et al. Hydrogen Sulfide Ameliorates Homocysteine-Induced Cardiac Remodeling and Dysfunction. Front Physiol 2019;10:598.

15. Lai WK, Kan MY. Homocysteine-Induced Endothelial Dysfunction. Ann Nutr Metab 2015;67:1-12.

16. Li W, Li Y, Cui S, et al. Se alleviates homocysteineinduced fibrosis in cardiac fibroblasts via downregulation of lncRNA MEG3. Exp Ther Med 2021;22:1269.

17. Han L, Tang Y, Li S, et al. Protective mechanism of SIRT1 on Hcy-induced atrial fibrosis mediated by TRPC3. J Cell Mol Med 2020;24:488-510. 
18. Boal F, Roumegoux J, Alfarano C, et al. Apelin regulates FoxO3 translocation to mediate cardioprotective responses to myocardial injury and obesity. Sci Rep 2015;5:16104.

19. Tan WQ, Wang K, Lv DY, et al. Foxo3a inhibits cardiomyocyte hypertrophy through transactivating catalase. J Biol Chem 2008;283:29730-9.

20. Sengupta A, Molkentin JD, Paik JH, et al. FoxO transcription factors promote cardiomyocyte survival upon induction of oxidative stress. J Biol Chem 2011;286:7468-78.

21. Norambuena-Soto I, Núñez-Soto C, Sanhueza-Olivares F, et al. Transforming growth factor-beta and Forkhead box $\mathrm{O}$ transcription factors as cardiac fibroblast regulators. Biosci Trends 2017;11:154-62.

22. Zhou Y, Richards AM, Wang P. Characterization and Standardization of Cultured Cardiac Fibroblasts for Ex Vivo Models of Heart Fibrosis and Heart Ischemia. Tissue Eng Part C Methods 2017;23:422-33.

23. Swynghedauw B. Molecular mechanisms of myocardial remodeling. Physiol Rev 1999;79:215-62.

24. Valiente-Alandi I, Potter SJ, Salvador AM, et al. Inhibiting Fibronectin Attenuates Fibrosis and Improves Cardiac Function in a Model of Heart Failure. Circulation 2018;138:1236-52.

25. Takawale A, Zhang P, Patel VB, et al. Tissue Inhibitor of Matrix Metalloproteinase-1 Promotes Myocardial Fibrosis by Mediating CD63-Integrin $\beta 1$ Interaction. Hypertension 2017;69:1092-103.

26. Xin Z, Ma Z, Hu W, et al. FOXO1/3: Potential suppressors of fibrosis. Ageing Res Rev 2018;41:42-52.

27. Nho RS, Hergert P. FoxO3a and disease progression. World J Biol Chem 2014;5:346-54.

28. Lee HY, You HJ, Won JY, et al. Forkhead factor, FOXO3a, induces apoptosis of endothelial cells through activation of matrix metalloproteinases. Arterioscler Thromb Vasc Biol 2008;28:302-8.

29. Wang C, Xu W, Zhang Y, et al. PARP1 promote autophagy in cardiomyocytes via modulating FoxO3a transcription. Cell Death Dis 2018;9:1047.

30. Pramod S, Shivakumar K. Mechanisms in cardiac fibroblast growth: an obligate role for Skp2 and FOXO3a in ERK1/2 MAPK-dependent regulation of p27kip1. Am J Physiol Heart Circ Physiol 2014;306:H844-55.

31. Yao L, Cao B, Cheng Q, et al. Inhibition of soluble epoxide hydrolase ameliorates hyperhomocysteinemiainduced hepatic steatosis by enhancing $\beta$-oxidation of fatty acid in mice. Am J Physiol Gastrointest Liver Physiol 2019;316:G527-38.

32. Chaturvedi P, Kamat PK, Kalani A, et al. High Methionine
Diet Poses Cardiac Threat: A Molecular Insight. J Cell Physiol 2016;231:1554-61.

33. Bárcena C, Quirós PM, Durand S, et al. Methionine Restriction Extends Lifespan in Progeroid Mice and Alters Lipid and Bile Acid Metabolism. Cell Rep 2018;24:2392-403.

34. Han L, Wu G, Feng C, et al. Dietary methionine restriction improves the impairment of cardiac function in middle-aged obese mice. Food Funct 2020;11:1764-78.

35. Frangogiannis NG. The Extracellular Matrix in Ischemic and Nonischemic Heart Failure. Circ Res 2019;125:117-46.

36. Frangogiannis NG. Cardiac fibrosis: Cell biological mechanisms, molecular pathways and therapeutic opportunities. Mol Aspects Med 2019;65:70-99.

37. Orogo AM, Gustafsson ÅB. Therapeutic targeting of autophagy: potential and concerns in treating cardiovascular disease. Circ Res 2015;116:489-503.

38. Qu J, Li M, Li D, et al. Stimulation of Sigma-1 Receptor Protects against Cardiac Fibrosis by Alleviating IRE1 Pathway and Autophagy Impairment. Oxid Med Cell Longev 2021;2021:8836818.

39. Seidenberg J, Stellato M, Hukara A, et al. The AP-1 Transcription Factor Fosl-2 Regulates Autophagy in Cardiac Fibroblasts during Myocardial Fibrogenesis. Int J Mol Sci 2021;22:1861.

40. Aminzadeh A, Mehrzadi S. Cardioprotective effect of levosimendan against homocysteine-induced mitochondrial stress and apoptotic cell death in H9C2. Biochem Biophys Res Commun 2018;507:395-9.

41. Zhao Q, Song W, Huang J, et al. Metformin decreased myocardial fibrosis and apoptosis in hyperhomocysteinemia -induced cardiac hypertrophy. Curr Res Transl Med 2021;69:103270.

42. Pattingre S, Tassa A, Qu X, et al. Bcl-2 antiapoptotic proteins inhibit Beclin 1-dependent autophagy. Cell 2005;122:927-39.

43. Aránguiz-Urroz P, Canales J, Copaja M, et al. Beta(2)adrenergic receptor regulates cardiac fibroblast autophagy and collagen degradation. Biochim Biophys Acta 2011;1812:23-31.

(English Language Editor: J. Jones)

Cite this article as: Shi Y, Zhao L, Zhang Y, Qin Q, Cong H, Guo Z. Homocysteine promotes cardiac fibrosis by regulating the Akt/FoxO3 pathway. Ann Transl Med 2021;9(23):1732. doi: 10.21037/atm-21-5602 


\section{Bright Field}

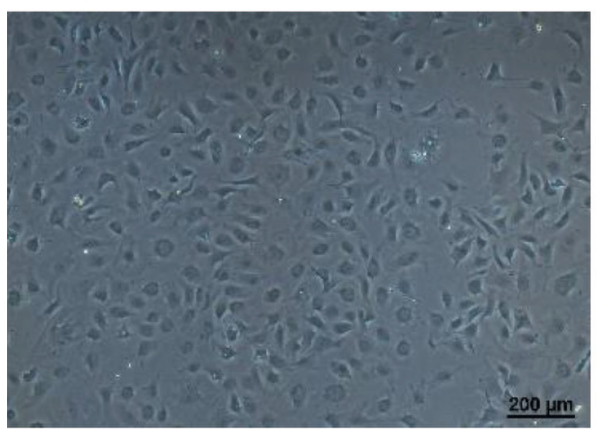

\section{DAPI/DDR2}

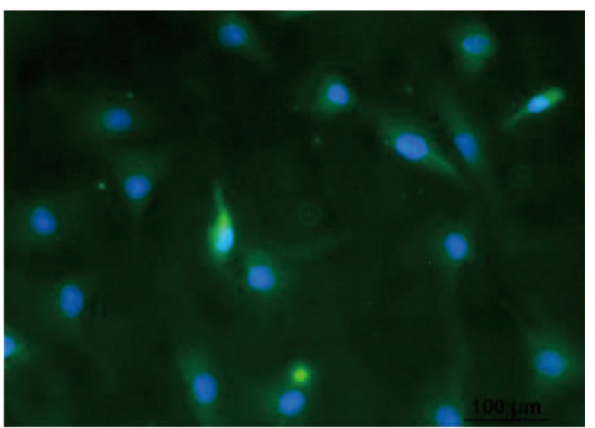

Figure S1 Characterization of cardiac fibroblasts. Brigt-field image of cultured cardiac fibroblasts (left) and representative immunostaining of discoidin domain receptor 2 (DDR2), a marker of cardiac fibroblasts. Scale bar, $200 \mu \mathrm{m}$ (left), $100 \mu \mathrm{m}$ (right).

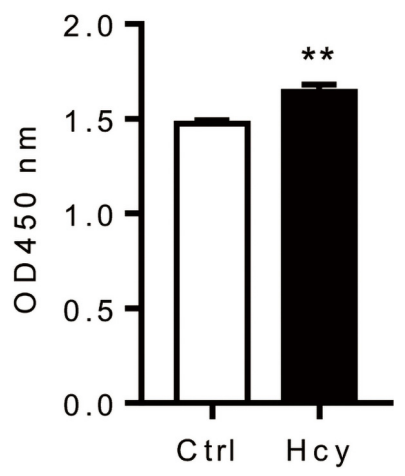

Figure S2 Hcy induced the proliferation of cardiac fibroblasts. Cardiac fibroblasts (CFs) were treated with homocysteine (Hcy) $(300 \mu \mathrm{M})$ for $24 \mathrm{~h}$, then added cck8 and detected the OD value at $450 \mathrm{~nm}$ after $2 \mathrm{~h}$ incubation. Data are shown as means \pm SEM, unpaired $\mathrm{t}$ test was used for comparisons. ${ }^{* *}, \mathrm{P}<0.01$.

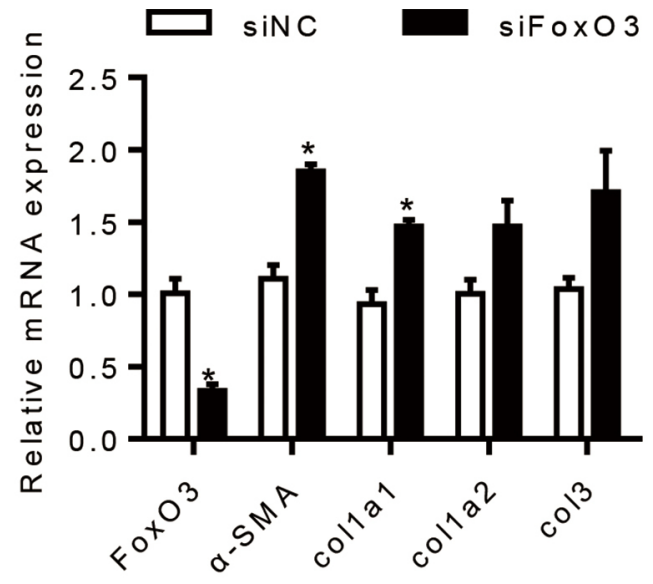

Figure S3 Knockdown FoxO3 promotes the differentiation of cardiac fibroblasts. Cardiac fibroblasts (CFs) were transfected with FoxO3 siRNA for $48 \mathrm{~h}$, then detected the mRNA levels. Data are shown as means \pm SEM, unpaired $t$-test was used for comparisons. *, $\mathrm{P}<0.05$. 